\title{
Un précurseur unique, quatre types de cellules intestinales
}

Au niveau de l'épithélium intestinal, quatre types cellulaires distincts assurent les fonctions de sécrétion (exocrine et endocrine), de transport et de défense de l'organisme : les entérocytes absorbants, les cellules à mucus, les cellules entéroendocrines et les cellules de Paneth (figure 1). L.es mécanismes moléculaires qui contrôlent la prolifération et la différenciation des cellules le long de l'axe cryptovillositaire sont encore énigmatiques. Il est aujourd'hui admis que les cellules souches indifférenciées de la crypte subissent une maturation au cours de leur migration vers la villosité au sommet de laquelle elles sont éliminées à l'état sénescent. En se divisant de façon asymétrique, chaque cellule souche donne naissance à une nouvelle cellule souche et à une cellule destinée à se différencier. L'exploration des mécanismes moléculaires conduisant au phénotype différencié a largement profité de l'étude d'un gène codant pour une protéine enzymatique spécifique de l'entérocyte, la sucrase-isomaltase (SI) [1]. Des travaux ont conduit à l'identification d'une région d'ADN du gène codant pour SI, capable de diriger spécifiquement l'expression d'un transgène dans l'épithélium intestinal et reconnaissant des facteurs de transcription exprimés spécifiquement dans l'intestin (protéines de la famille $\mathrm{Cdx}$ ) ou non spécifiques de l'intestin (comme HNF-1, hepalocyte nuclear fac(or-1) [2]. Si les mécanismes qui déterminent l'expression sélective du gène $S I$ dans l'entérocyte ne sont toujours pas élucidés, on apprend aujourd'hui dans une étude de transgenèse, que les quatre types cellulaires différenciés de l'épithélium possèdent la machinerie transcriptionnelle nécessaire à la synthèse de la protéine SI [3]. En outre, il est suggéré que l'absence d'expression de SI in vivo dans les cellules intestinales autres que les entérocytes serait due à un phénomène d'inhibition de la transcription du gène, processus complexe d'extinction génique qui pourrait être à l'origine du lignage cellulaire de l'épithélium digestif. Ainsi, afin d'analyser la spécificité tissulaire de la transcription du gène SI, des souris transgéniques et des lignées intestinales ont été utilisées. Un transgène a été construit à partir d'une séquence d'ADN élargie en 5' du gène $S I$ de souris $(-8,500$ à +54$)$ liée à un gène rapporteur codant pour l'hormone de croissance. L'expression du transgène chez les souris recombinantes est détectée majoritairement dans l'épithélium de l'intestin grêle. Dans les entérocytes, elle suit un gradient de concentration de la crypte vers la villosité qui coïncide parfaitement avec le gradient de concentration de l'ARNm de $S I$ endogène le long de cet axe. De façon surprenante, l'expression du transgène est également retrouvée dans les cellules entéroendocrines, les cellules à mucus et les cellules de Paneth, qui n'expriment pas SI in vivo. L.e transgène apparaît au jour 14 dans les cellules entéroendocrines, au jour 22 (période de sevrage) dans les entérocytes et à l'age adulte dans toutes les cellules de l'épithélium digestif, illustrant ainsi l'évolution de l'expression ectopique du transgène au cours du développement intestinal postnatal. I.'étude des mécanismes qui déterminent l'expression sélective d'un gène clans un type cellulaire spécifique a été poursuivie dans deux lignées de cellules intestinales, l'une de phénotype entérocytaire exprimant la protéine SI (Caco-2) et l'autre de phénotype entéroendocrine et n'exprimant pas de SI endogène (COL.O DM). Alors que la cellule Caco-2 synthétise une quantité importante d'ARNm codant pour SI, la cellule COI.O DM est incapable de synthétiser ce transcrit. I.e gène codant pour SI, bien que n'ayant subi aucune altération, n'est pas transcrit dans cette lignée. De façon surprenante, lorsque ces deux lignées sont transfectées avec un gène exogène contenant la région promotrice du gène $S I$, l'activité transcriptionnelle du promoteur SI est importante, voire supérieure, dans les cellules COI.O DM. Cela démontre, une nouvelle fois, que la cellule intestinale non entérocytaire a la potentialité et la machinerie transcriptionnelle nécessaire à la synthèse de SI, un mécanisme de répression du gène SI étant probablement responsable de l'absence de protéine SI endogène clans la lignée entéroendocrine COI.O DM. I a recherche de facteurs de transcription dirigeant l'expression de gènes spécifiques de l'intestin et participant donc au processus de développement et de différenciation de l'épithélium intestinal a conduit à considérer les produits des gènes $C d x l$ et $C d x 2$ comme étant les activateurs transcriptionnels des gènes exprimés spécifiquement dans l'intestin [4]. Les protéines $\mathrm{Cdx}$ font partie d'une famille de gènes homéotiques considérés, chez la Drosophile, comme des acteurs très précoces du développement. Chez la souris, ils semblent impliqués dans la formation de l'épithélium digestif au cours de l'embryogenèse. Récemment, la 


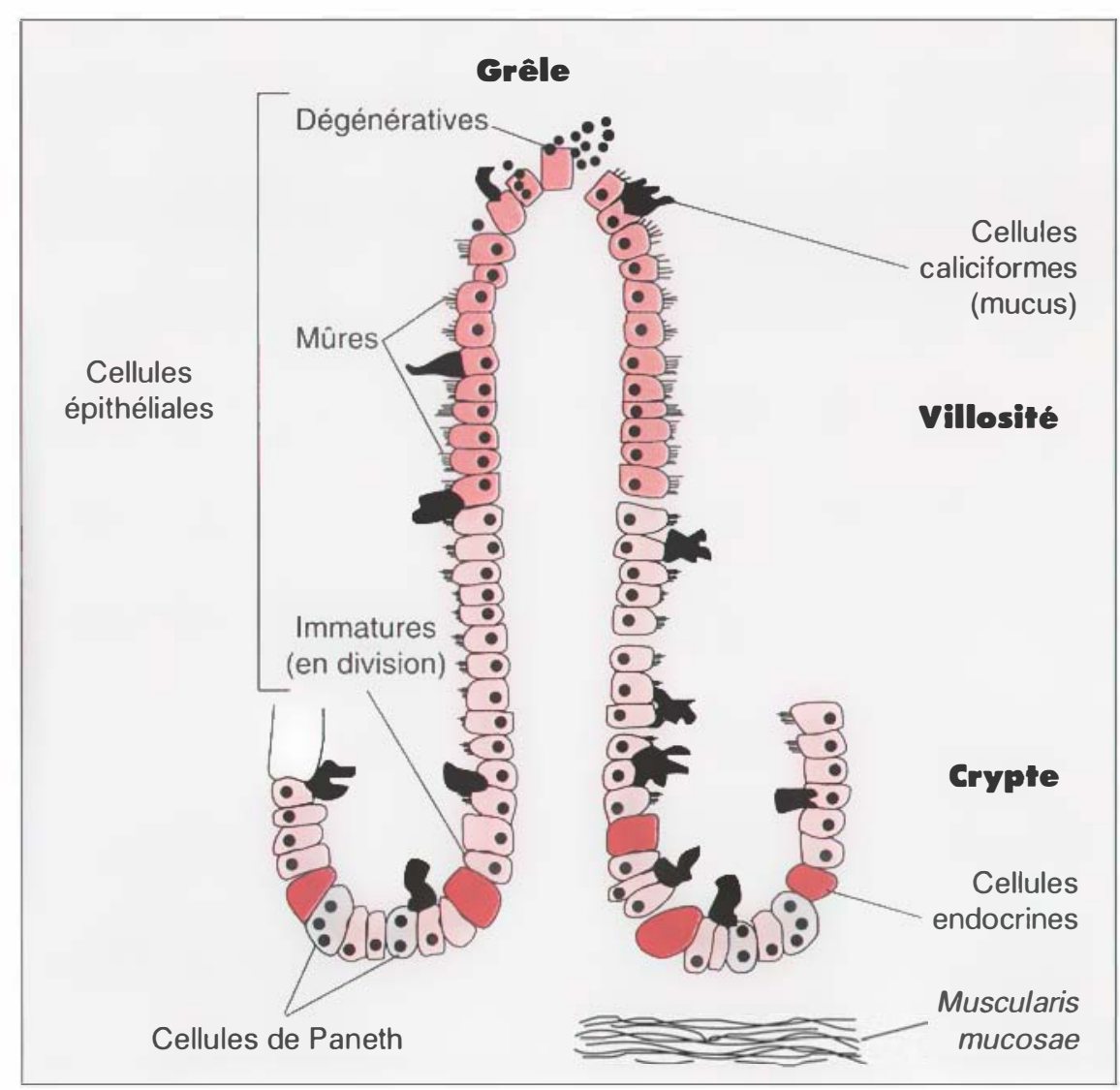

Figure 1. Description histologique de l'épithélium de l'intestin grêle. Les constituants cellulaires sont les cellules indifférenciées des cryptes, assurant le renouvellement cellulaire. Les cellules se déplacent vers le haut de la crypte, glissent sur les ponts intervillositaires et progressent vers le sommet de la villosité où elles sont éliminées à l'état sénescent. La migration s'accom pagne de maturation; 2) les cellules épithéliales mûres ou entérocytes absorbants, portant au pôle apical, une bordure en brosse ou microvillosités; 3) les cellules à mucus, dont la partie apicale est riche en grains de mucus. Le mucus intestinal joue un rôle protecteur de la muqueuse contre les substances luminales toxiques; 4) les cellules endocrines (en bistre) situées principalement au fond des cryptes, présentent des microvillosités au pôle apical et des grains de secrétions au pôle basal. Les principaux peptides sécrétés sont le GIP (gastric inhibitory polypeptide), la sécrétine, la cholecystokinine le peptide $Y Y$, le GLP-1 (glucagon-like peptide-1), I'entéroglucagon; 5) les cellules de Paneth (en gris), situées au fond des cryptes, possèdent de nombreux granules éosinophiles et un cytoplasme intensément basophile. Elles présentent une activité sécrétoire active riche en lysozymes. Leur cytoplasme comporte des immunoglobulines $A$ et $G$ qui leur confèrent un rôle de défense immunitaire. découverte de l'interaction de $\mathrm{Cdx} 2$ avec le promoteur minimal du gène SI (site désigné SIF1) et du rôle activateur de Cdx2 sur la transcription du gène $S I$, constituaient les premiers arguments en faveur de la participation de ce facteur de transcription dans les processus de différenciation intestinale [5]. Un travail réalisé in vitro par cette même équipe vient de confirmer la fonction de Cdx2 dans le développement et la différenciation intestinale [6]. La lignée IEC-6 a été établie à partir de cellules épithéliales dérivées d'un iléon de rat nouveau-né et présente un phénotype de cellules cryptiques indifférenciées (elles ne synthétisent pas de protéines $\mathrm{Cdxl}$ et $\mathrm{Cdx} 2$ endogènes). Les auteurs montrent que, après transfection stable d'une séquence d'ADN codant pour $\mathrm{Cdx} 2$, la prolifération et le phénotype des cellules IEC-6 sont totalement perturbés. Les cellules synthétisant la protéine $\mathrm{Cdx} 2$ ont une cinétique de prolifération complexe qui se caractérise par une inhibition de la croissance durant les trois premiers jours, suivie d'une croissance à vitesse "normale", aboutissant à une plus forte densité cellulaire à confluence. Contrastant avec les cellules d'origine poussant en monocouches, après 4 semaines de culture, les cellules synthétisant $\mathrm{Cdx} 2$ se présentent sous forme de structures multicellulaires arrangées en croisillons ; des entérocytes avec une bordure en brosse bien développée, quelques cellules à mucus et une matrice extracellulaire importante formée de fibres de collagène constituent la nouvelle architecture observée. Ce n'est qu'après 50 jours de culture, une fois les cellules différenciées, que l'ARNm codant pour SI est détecté dans les cellules exprimant $c d x 2$ exogène, suggérant ainsi que des événements tardifs sont impliqués dans la synthèse de cette enzyme. L'ensemble de ces données démontre que l'expression du gène $c d x 2$ dans les cellules intestinales non différenciées influence la prolifération, la morphogenèse et l'expression génique. Cdx2 est, à ce jour, le seul facteur de transcription connu pour participer au processus complexe de différenciation intestinale: il réglerait l'expression de gènes précoces impliqués 
dans la différenciation intestinale et de gènes tardifs nécessaires au maintien de cet état de différenciation. Même si on est encore loin d'avoir élucidé tous les phénomènes mis en œuvre dans le processus de différenciation intestinale, les résultats convergent tous vers la notion que la cellule souche des cryptes intestinales est à l'origine des différentes populations de cellules intestinales dans lesquelles coexistent des processus d'activation et de répression de gènes.

B.A.

1. Gordon JI, Hermiston MI.. Differentiation and self-renewal in the mouse gastrointestinal epithelium.Curn Op Ciell Biol 1994 ; 6 : 795-803

2. Markowitz AJ, W'u (ID), Birkenmeier EH, Traber PG. The human sucrase-isomaltase gene directs complex pattern of gene expression in transgenic mice. Am J Physiol (Gastrointest, Liver Physiol 28) $1993 ; 265:(.526-39$.

3. Markowitz AJ, Wu (SD), Bader A, Cui Z, Chen I., Traber PG. Regulation of lineage-specific transcription of the sucrase-isomaltase gene in transgenic mice and cell lines. Am I Physiol (Gasstrointest, liver Physiol 32) 1995 ; 269 : G925-39.

4. James R, Erler T, Kazenwadel J. Structure of the murine homeobox gene cdx-2. Fxpression in embryonic and adult intestinal epithelium. / Biol Chem $1994 ; 269: 15229$-37.

5. Traber $P(;, W u(;)$, Wang W. Novel DNA-binding proteins regulate intestine-specific transcription of the sucrase-isomaltase gene. Mol Ciell Biol $1992 ; 12: 3614-27$.

6. Sue E, Traber P(;. An intestine-specific homeobox gene regulates proliferation and differentiation. Mol Ciell Biol 1996; 16 : 619-25.

\section{MS ANWIVARGALRE EN VIDÉO-cagsentits}

Les conférences de la journée du $10^{\mathrm{C}}$ anniversaire de médecine/ sciences du 16 mars 1995 sont disponibles sur vidéo-cassettes auprès de :

\section{ASSOCIATION DIFFUSION DES CONNAISSANCES \\ 2, avenue Léon-Bernard. 35043 Rennes Cedex, France.}

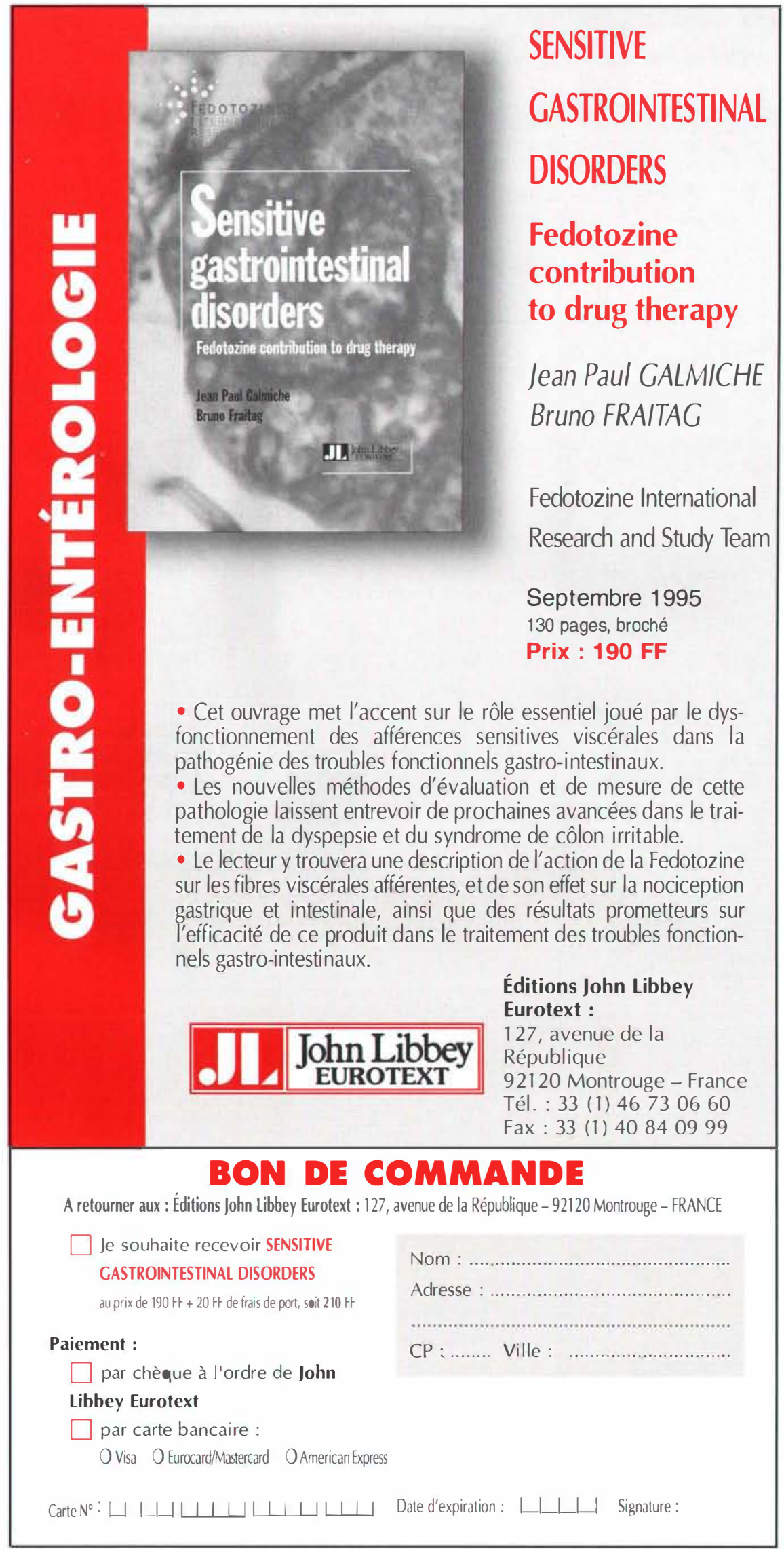

